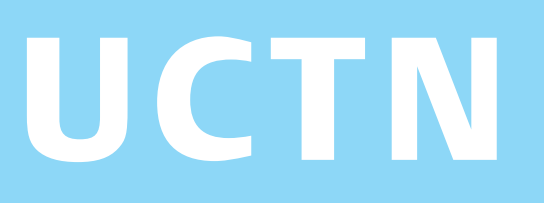

\title{
Diagnosis of multinodular goiter by endoscopic ultrasound-guided fine-needle aspiration
}

Endoscopic ultrasound-guided fine-needle aspiration (EUS-FNA) has been used to diagnose a variety of mediastinal masses, including lymphoma, neurogenic tumors, sarcoidosis, and malignant metastasis [1]. We report here a patient with a large superior mediastinal mass that was diagnosed as multinodular goiter by means of EUS-FNA.

A 67-year-old woman was admitted for resection of a colon cancer. A preoperative chest radiograph showed a right paratracheal mass (Figure $\mathbf{1}$ ), and computed tomography of the chest revealed a large multinodular goiter that extended into the mediastinum and a mediastinal mass extending retrosternally and contiguous with a large retrotracheal mass (Figure 2 ). Metastatic lymphadenopathy or a primary esophageal mass were considered in the differential diagnosis of this lesion in a patient with known colon cancer. Endoscopic ultrasound with a linear echo endoscope demonstrated a heterogeneous mass in the superior mediastinum, $5 \mathrm{~cm} \times 6 \mathrm{~cm}$ in size, with several anechoic areas, located $20 \mathrm{~cm}$ from the incisor teeth. EUS-FNA was performed using a 22-gauge needle, and two needle passes were made (Figure 3 ).

On-site cytopathological examination revealed cyst material, colloid with macrophages, and rare groups of benign follicular cells, findings consistent with cystic degeneration in a multinodular goiter (Figure 4). Because the patient was asymptomatic with respect to the mediastinal mass and because no malignancy was identified by EUS-FNA, the goiter was not investigated further and she underwent successful colectomy. At 12-month follow-up she remained asymptomatic and repeat computed tomography showed that there had been no change in the size of the goiter.

To our knowledge, there has only been one case of EUS-FNA of a thyroid mass reported in the literature [2]. In that report, a benign nodular goiter was diagnosed by EUS-FNA

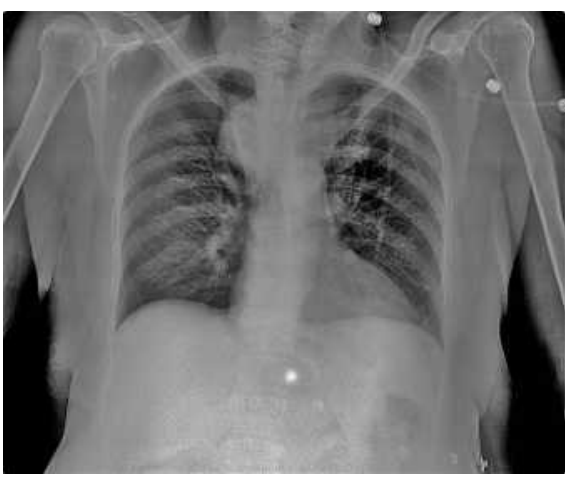

Figure 1 The preoperative chest radiograph in a patient with known colon carcinoma showed a large right paratracheal mass.
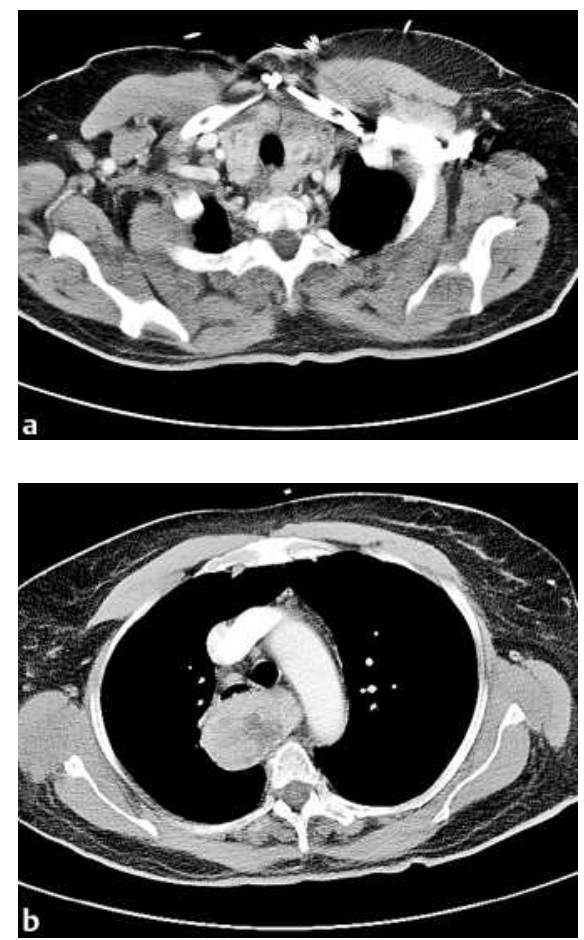

Figure 2 a Contrast-enhanced computed tomography of the chest revealed an enlarged, heterogeneous thyroid that extended into the mediastinum. $\mathbf{b}$ The inferior portion of the mass was significantly enlarged and appeared removed from the thyroid.

of a calcified superior mediastinal mass. In our patient, several anechoic areas were seen within the mediastinal mass at endoscopic ultrasound that were consistent with cystic degeneration. Fine-needle aspiration confirmed the diagnosis of goiter

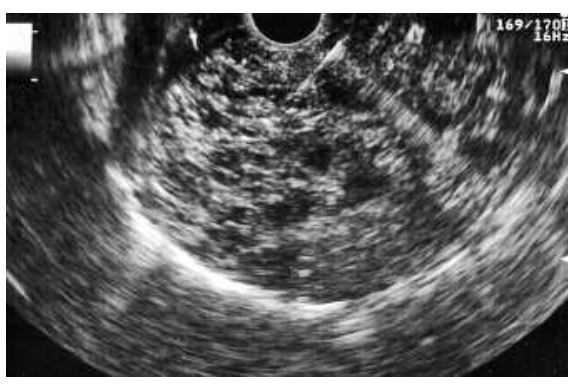

Figure 3 Endoscopic ultrasound view showing a heterogeneous mass measuring $5 \mathrm{~cm} \times 6 \mathrm{~cm}$ in the superior mediastinum with several anechoic areas, an appearance suggestive of cystic degeneration.
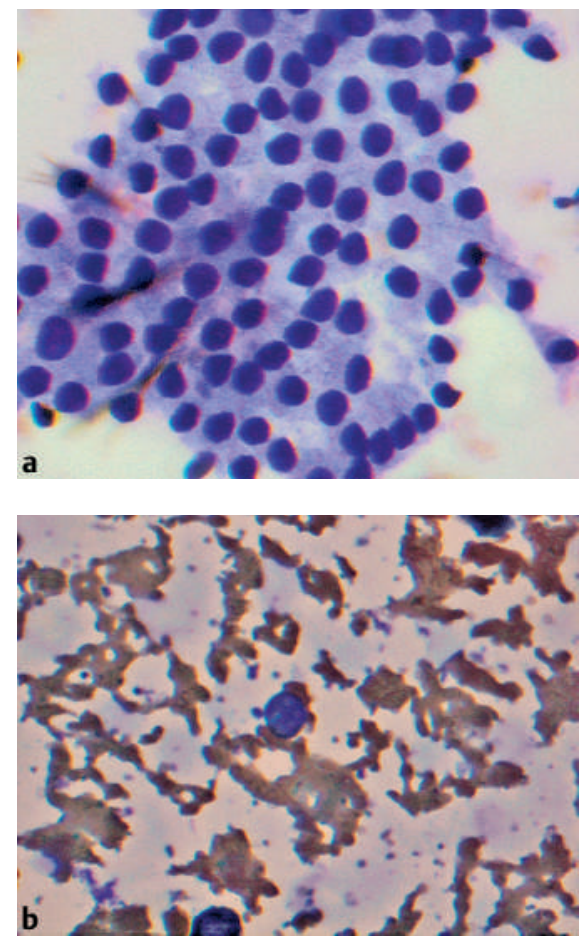

Figure 4 a Cytopathological examination showed follicular cells, consistent with a thyroid mass. $\mathbf{b}$ This appearance of a single pigment-laden macrophage on a background of colloid was consistent with a diagnosis of cystic degeneration in a multinodular goiter.

and excluded the presence of malignancy. Because EUS-FNA can be performed under conscious sedation, it is an attractive alternative to surgical or percutaneous sampl-

DOI: $10.1055 / s-2006-944711$ 
ing of accessible superior mediastinal lesions. In addition, EUS-FNA can be performed under direct, real-time ultrasound visualization. However, it is important to arrange follow-up of these patients because a small tumor within the goiter could be missed by EUS-FNA.

Endoscopy_UCTN_Code_CPL_1AL_2AC

\section{S. Varadarajulu1, D. N. Jhala ${ }^{2}$, C. L. Canon ${ }^{3}$}

${ }^{1}$ Division of GastroenterologyHepatology, University of Alabama at Birmingham School of Medicine, Birmingham, Alabama, USA
2 Department of Pathology, University of Alabama at Birmingham School of Medicine, Birmingham, Alabama, USA

${ }^{3}$ Department of Radiology, University of Alabama at Birmingham School of Medicine, Birmingham, Alabama, USA.

\section{References}

${ }^{1}$ Panelli F, Erickson RA, Prasad VM. Evaluation of mediastinal masses by endoscopic ultrasound and endoscopic ultrasound-guided fine-needle aspiration. Am J Gastroenterol 2001; 96: $401-408$

${ }^{2}$ DeWitt J, Youssef W, LeBlanc J et al. EUSguided FNA of a thyroid mass. Gastrointest Endosc 2004; 59: $307-310$
Corresponding Author

\section{S. Varadarajulu, M.D.}

Division of Gastroenterology-Hepatology University of Alabama at Birmingham Medical Center

410 LHRB, 1530 3rd Ave. S.

Birmingham

Alabama 35294

USA

Fax: $\quad+1-205-975-6381$

E-mail: svaradarajulu@yahoo.com 